\title{
TWO NEW EQUIVALENT STATEMENTS TO RIEMANN HYPOTHESIS
}

\section{Jamal Salah}

Department of Basic Science

College of Applied and Health Sciences

A'Sharqiyah University

Ibra, Oman

e-mail: damous73@yahoo.com

\begin{abstract}
The distribution of such prime numbers among all natural numbers does not follow any regular pattern; however, the German mathematician G. F. B. Riemann (1826-1866) observed that the frequency of prime numbers is very closely related to the behavior of an elaborate function called the Riemann zeta function $\zeta(s)$. The nontrivial zeroes of zeta function have $1 / 2$ as their real part. This has been checked for the first $1,500,000,000$ solutions. A proof that it is true for every interesting solution would shed light on many of the mysteries surrounding the distribution of prime numbers. The celebrated Riemann hypothesis remains unsolved since it was introduced in 1859. Miscellaneous approaches have been considered without any exact and complete proof. Furthermore, some equivalent statements have been established.

In this work, we consider the famous Robin inequality and propound a connection to the theory of univalent functions by the means of Koebe function.
\end{abstract}

Received: June 30, 2019; Revised: July 13, 2019; Accepted: July 15, 2019

2010 Mathematics Subject Classification: 30C45, 30C50.

Keywords and phrases: Riemann hypothesis, Robin's inequality, the theory of univalent functions, fractional calculus. 


\section{Introduction and Statement of the Problem}

Identified as one of the 7 Millennium Problems, see [1, 2, 4-9, 19, 33-35], the Riemann zeta hypothesis has successfully evaded mathematicians for over 100 years. Simply stated, Riemann conjectured that all of the nontrivial zeroes of the zeta function have real part equal to $1 / 2$. In his attempt to provide an accurate gap between prime numbers, Riemann has considered the Euler's combination of $p$-series and prime numbers:

$$
\sum_{n=1}^{\infty} n^{-s}=\prod_{p-\text { prime }} \frac{1}{1-p^{-s}}, \quad s>1 .
$$

The left hand side of the equation above is simply called zeta function, which is convergent for $s>1$. Riemann implemented complex analysis in order to expand the convergence by the means of complex field, i.e., he considered $s$ as a complex number and provided an entire expansion by the means of Gamma function as follows:

$$
\zeta(s)=\sum_{n=1}^{\infty} n^{-s}=\int_{0}^{\infty} \frac{1}{1-e^{-t}} t^{s-1} d t
$$

The above function is entire except a simple hole at $s=1$. Furthermore, trivial zeros of the zeta function could be readily provided by the functional equations (see [1]). However, the non-trivial zeroes that Riemann has conjectured to lie on the critical strip and with real part equal to $1 / 2$ is what we call Riemann hypothesis (RH), simply saying:

If $s$ is a zero of zeta function, then $\operatorname{Re}(s)=1 / 2$.

\section{Robin's Inequality}

Since it was introduced, RH inspired a lot of mathematicians to decode its ambiguity by the means of miscellaneous approach either through complex analysis, analytical number theory or even by elementary approach. Many equivalent statements to $\mathrm{RH}$ have been introduced which provide 
alternative strategies and methodologies. References ([3] and [36]) provided a beautiful equivalent inequality to RH. Robin's inequality states that:

$\mathrm{RH}$ is equivalent to:

$$
\frac{\sigma(n)}{n}<e^{\gamma} \ln \ln (n) \text { for } n>5041 \text { and } \gamma=0.57 \ldots \text { (Euler's constant). }
$$

Since then, some partial results have been proved, see, for example, $[6,7]$. Mathematically, despite the huge number of zeta zeros that have $1 / 2$ as real part, none can assume that RH is proved. Unfortunately, all attempts to prove (disprove) $\mathrm{RH}$ remain incomplete, that is a different approach which may be considered in the future. Recently, in 2017, Durmagambetov [7] introduced a modified zeta function which allows the estimation of Riemann's zeta function and solving Riemann problem. Some authors used analytic method to deny the Riemann hypothesis [9]. Wünsche approached to Riemann hypothesis by means of second mean value theorem of calculus. However, our motivation towards Riemann problem is to involve fractional calculus through univalent functions, some advancement made in this connection can be studied in [5] and [6].

\section{New Approach}

The aim of this piece of work is to implement the theory of univalent functions in $\mathrm{RH}$ in accordance its wide connection with fractional calculus applications. The difficulty certainly arises from the chaotic and unpredictable pattern of the sum of divisors function. With a numerical approach and by manipulating the tolerance error, one may possibly wish to partially prove RH. This is due to the wide applications of fractional calculus that implies a crucial impact on the univalent functions along with its miscellaneous applications in mathematics, physics and economy, see [10-17, 22]. 


\section{Main Propositions}

In order to derive the main results, we need to apply the following lemmas. Further definitions and relevant results on special and univalent functions can be found in [18, 20, 21, 23-32].

Lemma 1. Given that $f(z)$ of the form: $f(z)=z+\sum_{n=2}^{\infty} a_{n} z^{n}$ is univalent, then:

$$
\left|a_{n}\right| \leq n, \text { for every } n=2,3,4, \ldots \text { and }|z|<1 .
$$

Lemma 2. Given that $f(z)$ of the form: $f(z)=z+\sum_{n=2}^{\infty} a_{n} z^{n}$ is convex, then:

$$
\left|a_{n}\right| \leq 1, \text { for every } n=2,3,4, \ldots \text { and }|z|<1 .
$$

Next, two new criteria or statements that are equivalent to $\mathrm{RH}$ are readily proven:

\section{Theorem 1.}

$$
R H \text { is equivalent to: } f(z)=z+\sum_{n=5041}^{\infty} \frac{\sigma(n)}{e^{\gamma} \ln \ln (n)} z^{n} \text { is univalent. }
$$

Proof. For $f(z)=z+\sum_{n=2}^{\infty} a_{n} z^{n}$, let $a_{n}=\frac{\sigma(n)}{e^{\gamma} \ln \ln (n)}$ and $a_{i}=0$, $i=2,3,4, \ldots, 5040$. If $f(z)$ is univalent, then by Lemma $1, a_{n}=$ $\frac{\sigma(n)}{e^{\gamma} \ln \ln (n)}<n, \quad n \geq 5041$, that is Robin inequality which is equivalent to Riemann hypothesis.

\section{Theorem 2.}

$R H$ is equivalent to: $g(z)=z+\sum_{n=5041}^{\infty} \frac{\sigma(n)}{e^{\gamma} n \ln \ln (n)} z^{n}$ is convex. 
Proof. For $g(z)=z+\sum_{n=2}^{\infty} a_{n} z^{n}$, let $a_{n}=\frac{\sigma(n)}{e^{\gamma} n \ln \ln (n)}$ and $a_{i}=0$, $i=2,3,4, \ldots, 5040$. If $f(z)$ is convex, then by Lemma $2, a_{n}=$ $\frac{\sigma(n)}{e^{\gamma} n \ln \ln (n)}<1, n \geq 5041$, that is Robin inequality which is equivalent to Riemann hypothesis.

The assertions above follow from the coefficient bound of univalent (convex) functions.

\section{Plausible Approach (Open Problems)}

In order to achieve the objective, we consider the following steps:

(a) Given $f(z)=z+\sum_{n=1}^{\infty} a_{n} z^{n}$ is univalent, if $a_{i}=0$ for some $i=1,2,3, \ldots$ is $f(z)$ still univalent?

(b) Given $g(z)=z+\sum_{n=1}^{\infty} b_{n} z^{n}$ is convex, if $b_{i}=0$ for some $i=1,2,3, \ldots$ is $g(z)$ still convex?

(c) Given $f(z)=z+\sum_{n=1}^{\infty} a_{n} z^{n}$. How to derive the general form of $f(z)$ without using Taylor series?

(d) Curve fitting $(n: \sigma(n))$ by the means of convenient methods like Newton's, Lagrange interpolating and by using computer process.

(e) Assuming that the function generated in (d) represents $f(z)$ and $g(z)$ by considering and manipulating the error.

(f) Use graphics to show the univalency (convexity).

(g) Control the error such that the generated functions satisfy Robin's condition. 
Jamal Salah

\section{Acknowledgement}

The author thanks the anonymous referees for their valuable suggestions and comments which improved the presentation of the paper.

\section{References}

[1] Arthur M. Jaffe, The millennium grand challenge in mathematics, Notices Amer. Math. Soc. 53(6) (2000), 652-660.

[2] J. Lagarias, An elementary problem equivalent to the Riemann hypothesis, Amer. Math. Monthly 109 (2002), 534-543.

[3] G. Robin, Grandes valeurs de la fonction Somme des diviseurs et hypothese de Riemann, J. Math. Pures Appl. 63 (1984), 187-213.

[4] E. Bombieri, Problems of the Millennium: The Riemann Hypothesis, http://www.claymath.org/millennium/Riemann_Hypothesis/Official_Problem_De scription.pdf.

[5] Jamal Salah, On Riemann hypothesis and Robin's inequality, International Journal of Scientific and Innovative Mathematical Research (IJSIMR) 3(4) (2015), 9-14.

[6] Young Ju Choie, Nicolas Lichiardopol, Pieter Moree and Patrick Solé, On Robin's criterion for the Riemann hypothesis, J. Théor. Nombres Bordeaux 19(2) (2007), 357-372.

[7] A. A. Durmagambetov, Riemann hypothesis, Journal of Applied Mathematics and Physics 5 (2017), 1424-1430.

[8] Jinua Fei, About the Riemann hypothesis, Journal of Applied Mathematics and Physics 4 (2016), 561-570.

[9] Alfred Wünsche, Approach to a proof of the Riemann hypothesis by the second mean-value theorem of calculus, Advances in Pure Mathematics 6 (2016), 972-1021.

[10] Jamal Y. Salah, A new subclass of univalent functions defined by the means of Jamal operator, Far East J. Math. Sci. (FJMS) 108(2) (2018), 389-399. http://dx.doi.org/10.17654/MS108020389.

[11] Hameed Ur Rehman, Maslina Darus and Jamal Salah, A note on Caputo's derivative operator interpretation in economy, J. Appl. Math. 2018, Art. ID 1260240, 7 pp. DOI: 10.1155/2018/1260240. 
[12] Hameed Ur Rehman, Maslina Darus and Jamal Salah, Normalization of the generalized $K$-Mittag-Leffler function and ratio to its sequence of partial sums, BSG Proceedings, Vol. 25, 2018, pp. 78-94.

[13] Hameed Ur Rehman, Maslina Darus and Jamal Salah, Graphing examples of starlike and convex functions of order $\beta$, Appl. Math. Inf. Sci. 12(3) (2018), 509-515.

[14] Hameed Ur Rehman, Maslina Darus and Jamal Salah, Coefficient properties involving the generalized $K$-Mittag-Leffler functions, Trans. J. Math. Mech. (TJMM) 9(2) (2017), 155-164.

[15] Jamal Y. Salah, A note on the Hurwitz zeta function, Far East J. Math. Sci. (FJMS) 101(12) (2017), 2677-2683.

[16] Jamal Y. Salah, Closed-to-convex criterion associated to the modified Caputo's fractional calculus derivative operator, Far East J. Math. Sci. (FJMS) 101(1) (2017), 55-59.

[17] Jamal Y. Salah, Properties of the modified Caputo's derivative operator for certain analytic functions, Int. J. Pure Appl. Math. 109(3) (2016), 665-671.

[18] Jamal Salah, A note on the modified Caputo's fractional calculus derivative operator, Far East J. Math. Sci. (FJMS) 100(4) (2016), 609-615.

[19] Jamal Salah, A note on Riemann zeta function, IOSR Journal of Engineering (IORS-JEN) 6(2) (2016), 7-16.

[20] Jamal Salah, Neighborhood of a certain family of multivalent functions with negative coefficients, Int. J. Pure Appl. Math. 92(4) (2014), 591-597.

[21] Jamal Salah, A note on gamma function, International Journal of Modern Sciences and Engineering Technology (IJMSET) 2(8) (2015), 58-64.

[22] Jamal Salah and Venkatesh Srivastava, Inequalities on the theory of univalent function, Journal of Mathematics and System Science 4 (2014).

[23] Jamal Salah, Fekete-Szego problems involving certain integral operator, International Journal of Mathematics Trends and Technology (IJMTT) 7(1) (2014), 54-60.

[24] Jamal Salah, Subordination and superordination involving certain fractional operator, Asian Journal of Fuzzy and Applied Mathematics 1 (2013), 98-107.

[25] Jamal Salah and Maslina Darus, On convexity of the general integral operators, An. Univ. Vest Timis. Ser. Mat.-Inform. 49(1) (2011), 117-124. 
[26] Jamal Salah and Maslina Darus, A note on generalized Mittag-Leffler function and applications, Far East. J. Math. Sci. (FJMS) 48(1) (2011), 33-46.

[27] Jamal Salah, Certain subclass of analytic functions associated with fractional calculus operator, Trans. J. Math. Mech. (TJMM) 3 (2011), 35-42.

[28] Jamal Salah, A note on starlike functions of order $\alpha$ associated with a fractional calculus operator involving Caputo's fractional, J. Appl. Comp. Sci. Math. 5 (2011), 98-101.

[29] Jamal Salah and Maslina Darus, The univalency of certain analytic integral operator, UKM Mathematic Post Graduate Workshop, 2009.

[30] Jamal Salah and Maslina Darus, A subclass of uniformly convex functions associated with a fractional calculus operator involving Caputo's fractional differentiation, Acta. Univ. Apulensis Math. Inform. 24 (2010), 295-306.

[31] V. E. Tarasov, Interpretation of fractional derivatives as reconstruction from sequence of integer derivatives, Fund. Inform. 151(1-4) (2017), 431-442.

[32] V. V. Tarasova and V. E. Tarasov, Economic interpretation of fractional derivatives, Progress in Fractional Differentiation and Applications 3(1) (2017), $1-7$.

[33] E. C. Titchmarsh, The Theory of the Riemann Zeta-function, Oxford Science Publications, Second Edition, Revised by D. R. Heath-Brown, 1986.

[34] E. Artin, The gamma function, Translated by Michael Butler, Athena Series: Selected Topics in Mathematics, Holt, Rinehart and Winston, New York, 1964.

[35] G. H. Hardy and E. M. Wright, An Introduction to the Theory of Numbers, D. R. Heath-Brown and J. H. Silverman, eds., 6th ed., Oxford University Press, Oxford, 2008.

[36] S. Ramanujan, Highly composite numbers, Annotated and with foreword by J.-L. Nicolas and G. Robin, Ramanujan J. 1(2) (1997) 119-153. 